\title{
Active beamforming with interpolated FIR filtering
}

\author{
P. P. Vaidyanathan and Ching-Chih Weng \\ Dept. of Electrical Engineering, 136-93 \\ California Institute of Technology, Pasadena, CA 91125, USA \\ ppvnath@systems.caltech.edu, cweng@caltech.edu
}

\begin{abstract}
The interpolated FIR (IFIR) radar was recently introduced in the context of MIMO radar theory. It was shown that this system has a signal to clutter ratio intermediate between those of the SIMO and MIMO radars. This paper considers the optimal design of the active IFIR beamformer in presence of jammers. It is shown that this beamformer can achieve beamwidths as sharp as those of colocated MIMO radars with full-length virtual arrays. At the same time, the extra complexity of MIMO radars, which arises from use of multiple transmitter waveforms and several sets of receiver matched filter banks, is not present in the IFIR realization. Design examples for IFIR radars which optimize the receiver beamforming weights in presence of jammers for fixed transmitter are also presented. ${ }^{1}$
\end{abstract}

Index Terms-Beamforming, IFIR radar, MIMO radar.

\section{INTRODUCTION}

$\mathbf{I}^{\mathrm{r}}$ $\mathrm{N}$ recent years the MIMO radar has received considerable attention [1]-[11]. One of its advantages is that it can be configured in such a way that the receiver functions as a virtual array with $N_{t} N_{r}$ virtual antenna elements, where $N_{t}$ and $N_{r}$ are the number of antenna elements in the transmitting and receiving uniform linear arrays (ULAs.) The additional number of freedoms offered by the virtual array can be exploited in a number of ways. One of these is that the transmit-receive beam pattern can be designed to be much sharper than that of a traditional phased array radar (also called SIMO radar). It is known that the MIMO radar provides an improved signal to clutter ratio at the receiver (compared to a SIMO radar) although the signal to noise ratio can get worse [11]. Thus in a clutter dominated scenario, the MIMO radar has some advantages.

An alternative to MIMO radar is the so-called interpolated FIR or IFIR radar introduced in [11]. This system is simpler to implement, as it avoids the multiple waveforms at the transmitter used by MIMO radars, and therefore avoids the set of matched filter banks (Sec. II) required at the receiver of the MIMO radar. Furthermore, it has been shown in [11] that the IFIR radar also provides an improvement in the signal to clutter ratio, although not as much as a MIMO radar does.

In this paper we focus on the beamforming aspects and show that if sharpness of the transmit-receive beam is of main interest then the IFIR radar is an excellent compromise to the MIMO radar. Sec. II reviews the SIMO, MIMO, and IFIR radars briefly. In Sec. III we describe optimal ways to design the beamforming coefficients of the IFIR radar in the

\footnotetext{
${ }^{1}$ This work was supported in parts by the ONR grant N00014-08-1-0709, and the California Institute of Technology.
}

presence of jammers. These methods provide very sharp beams compared to SIMO radars of the same complexity. Design examples are included to demonstrate the ideas.

\section{THE IFIR BEAMFORMER}

Fig. 1 shows the uniform linear arrays (ULAs) of the transmitter and receiver in a radar. We assume these are colocated so that the angle $\theta$ as seen by a target is identical at the transmitter and receiver. This system is said to be a SIMO radar if $d_{t}=d_{r}$, and the waveforms $s_{k}(t)$ are all identical. It is said to be a MIMO radar if $s_{k}(t)$ are orthogonal, i.e., $\int s_{k}(t) s_{m}^{*}(t) d t=\delta[k-m]$. In the MIMO radar each of the $N_{r}$ receiving sensors is followed by a matched filter bank, with $N_{t}$ matched filters, one matched to each waveform $s_{k}(t)$ (Fig. 2 ). With this choice, and with the element spacings chosen such that $d_{t}=N_{r} d_{r}$, it can be shown [2] that the target and clutter essentially see a virtual receiver array with $N_{t} N_{r}$ elements spaced apart by $d_{r}$ (part (b) of Fig. 2). In a SIMO radar the transmitter and receiver beams have the form

$$
H\left(e^{j \omega}\right)=\sum_{n=0}^{N_{t}-1} a_{n} e^{-j \omega n}
$$

and

$$
G\left(e^{j \omega}\right)=\sum_{n=0}^{N_{r}-1} b_{n} e^{-j \omega n}
$$

where $\omega=2 \pi d \sin \theta / \lambda, \theta$ is the angle with respect to vertical (Fig. 1), and $\lambda$ is the wavelength of the signal, assumed to be narrowband.

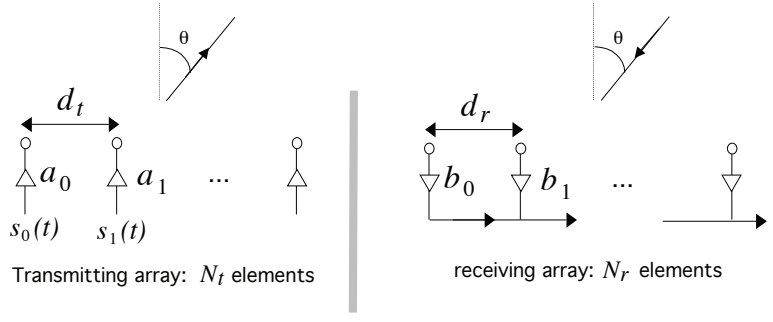

Fig. 1. The transmitter and receiver arrays in an active beamformer.

In a MIMO radar there is no beam forming at the transmitter since $s_{k}(t)$ are orthogonal (noncoherent). The virtual array at 

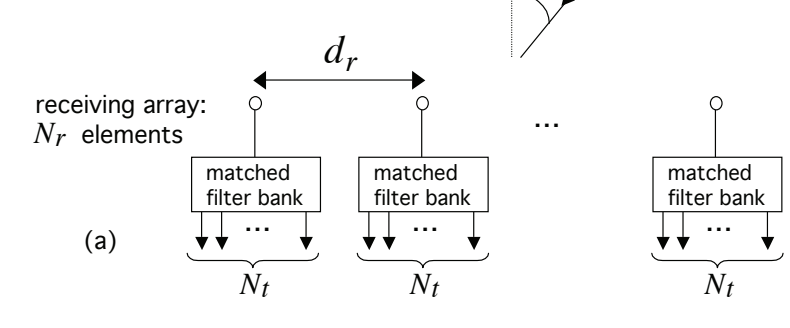

(b)

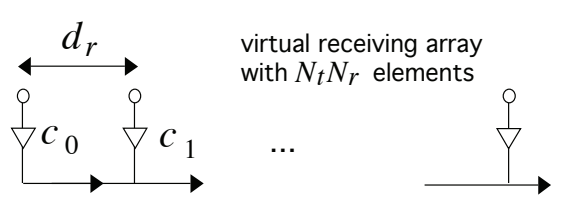

Fig. 2. (a) The MIMO radar receiver, with a bank of $N_{t}$ matched filters at the output of each of the $N_{r}$ sensors. (b) The virtual array generated by the MIMO radar.

the receiver forms the beam

$$
H_{v}\left(e^{j \omega}\right)=\sum_{n=0}^{N_{t} N_{r}-1} c_{n} e^{-j \omega n}
$$

Since this has $N_{t} N_{r}$ taps, the beam can be much narrower and can also have smaller side lobes compared to the transmitreceive beam $H\left(e^{j \omega}\right) G\left(e^{j \omega}\right)$ of the SIMO radar.

Next, the IFIR radar or beamformer is such that $s_{k}(t)=s(t)$ as in a SIMO radar. However the element spacing is chosen as in a MIMO radar, that is $d_{t}=N_{r} d_{r}$. With $d_{r}=\lambda / 2$ where $\lambda$ is the wavelength of the narrowband signal, the transmitted beam has the form

$$
\sum_{n=0}^{N_{t}-1} a_{n} e^{-j N_{r} \omega n}=H\left(e^{j \omega N_{r}}\right)
$$

This has $N_{r}$ main lobes. Of these one of them, say the one centered around $\omega=0$ (i.e., $\theta=0$ ), is the desired main lobe and the $N_{r}-1$ extra copies are the undesirable grating lobes. In the IFIR beamformer these extra lobes are simply suppressed by the receiver beam $G\left(e^{j \omega}\right)$. This is similar in principle to the design of IFIR digital filters [12], [13], as demonstrated in Fig. 3 . Here the filter $H\left(e^{j \omega}\right)$ has one passband in $[-\pi, \pi)$, whereas $H\left(e^{j \omega N_{r}}\right)$ has $N_{r}$ passbands separated by $2 \pi / N_{r}$. The filter $G\left(e^{j \omega}\right)$ retains one of these $N_{r}$ passbands. The result is a narrow band filter $H\left(e^{j \omega N_{r}}\right) G\left(e^{j \omega}\right)$, at a low cost equal to that of the broadband filters $H\left(e^{j \omega}\right)$ and $G\left(e^{j \omega}\right)$. This is what motivated the introduction of name "IFIR radar" in [11].

Figure 4 shows the element spacing and beams in SIMO radar (part (a)), the virtual array and sharper beam in MIMO radar (part (b)), and the transmitter array and its beam in IFIR radar (part (c)). The composite beam $H\left(e^{j \omega N_{r}}\right) G\left(e^{j \omega}\right)$ has a $N_{r}$-times sharper main lobe compared to the SIMO composite beam $H\left(e^{j \omega}\right) G\left(e^{j \omega}\right)$. Thus the IFIR radar can achieve the same beam sharpness as a MIMO radar but without the added complexity of multiple waveforms and matched filter banks. The number of beam-forming freedoms in an IFIR radar is $N_{t}+N_{r}$ (i.e., the $a_{n}$ 's and $b_{n}$ 's) as in a SIMO radar, and is much smaller than the $N_{t} N_{r}$ freedoms of the virtual array of a MIMO radar. However, if the main goal is the sharpness of the main beam, then the IFIR method offers a simple way to achieve it.

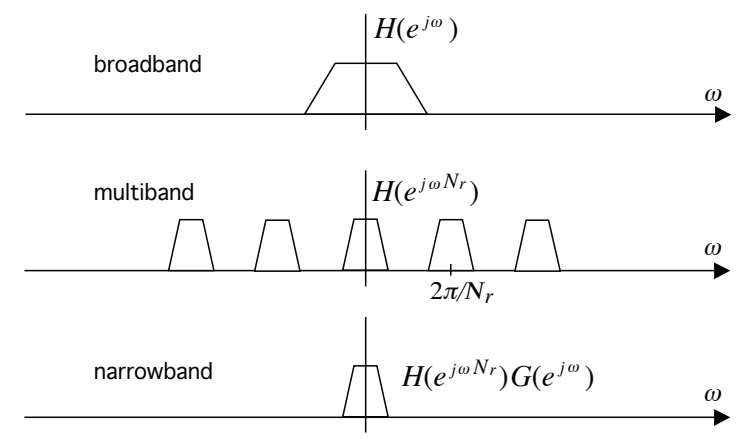

Fig. 3. Explanation of how the interpolated FIR (IFIR) filter works. See text.

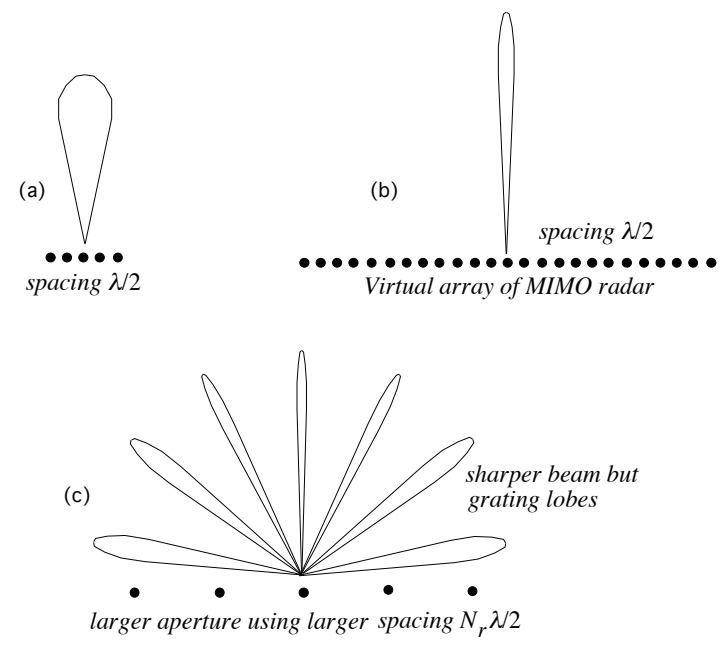

Fig. 4. Summary of beamforming using different methods. See text.

\section{OPTIMIZATION OF IFIR RADAR COEFFICIENTS}

In this section we consider the optimization of the beamforming coefficients $b_{n}$ at the receiver of the IFIR radar. We present two such examples, which have two different types of transmitter beamforming.

Example 1. In the first example we consider $N_{t}=5, N_{r}=$ $10, d_{t}=N_{r} d_{r}$, and $d_{r}=\lambda / 2$. The transmitting beamformer is a simple phased array with $a_{n}=1$ for $0 \leq n \leq N_{t}-1$. The weights $b_{n}$ for the receiving array are obtained using the minimum-variance distortionless response (MVDR) approach 
[15]. Two jammers are assumed, at the angles $\theta=45^{\circ}$ and $-30^{\circ}$ respectively. Thus the autocorrelation of the received signal has the form

$$
\mathbf{R}=\sigma_{s}^{2} \mathbf{s s}^{\dagger}+\sigma_{j_{1}}^{2} \mathbf{s}_{j_{1}} \mathbf{s}_{j_{1}}^{\dagger}+\sigma_{j_{2}}^{2} \mathbf{s}_{j_{2}} \mathbf{s}_{j_{2}}^{\dagger}+\sigma_{n}^{2} \mathbf{I}
$$

where $\mathbf{s}$ is the signal steering vector (equal to $\left[\begin{array}{ll}1 & 1 \ldots 1\end{array}\right]^{T}$ in this example), and $\mathbf{s}_{j_{1}}$ and $\mathbf{s}_{j_{2}}$ are the $N_{t}$-element jammer steering vectors: $\mathbf{s}_{j_{k}}=\left[\begin{array}{llll}1 & e^{-j \omega_{j_{k}}} & e^{-j 2 \omega_{j_{k}}} & \ldots\end{array}\right]^{T}$. We assume the noise level is $-20 \mathrm{~dB}$ (i.e., $\sigma_{n}^{2} / \sigma_{s}^{2}$ is $-20 \mathrm{~dB}$ ) and the jammer levels are $10 \mathrm{~dB}$ and $0 \mathrm{~dB}$ (i.e., $\sigma_{j_{k}}^{2} / \sigma_{s}^{2}$ are $10 \mathrm{~dB}$ and $0 \mathrm{~dB}$ ). Clutter is ignored in this example. The MVDR problem is to minimize the mean square value of the receiving beamformer output, which is $\phi=\mathbf{b}^{\dagger} \mathbf{R b}$, subject to the constraint $\mathbf{b}^{\dagger} \mathbf{s}=1$. Here $\mathbf{b}=\left[\begin{array}{lll}b_{0} & b_{1} & \ldots \\ b_{N_{r}-1}\end{array}\right]^{T}$. Since

$\mathbf{b}^{\dagger} \mathbf{R} \mathbf{b}=\sigma_{s}^{2} \mathbf{b}^{\dagger} \mathbf{s} \mathbf{s}^{\dagger} \mathbf{b}+\sigma_{j_{1}} \mathbf{b}^{\dagger} \mathbf{s}_{j_{1}} \mathbf{s}_{j_{1}}^{\dagger} \mathbf{b}+\sigma_{j_{2}} \mathbf{b}^{\dagger} \mathbf{s}_{j_{2}} \mathbf{s}_{j_{2}}^{\dagger} \mathbf{b}+\sigma_{n}^{2} \mathbf{b}^{\dagger} \mathbf{b}$, minimizing $\phi$ is equivalent to minimizing the sum of the last three terms (because the first term reduces to a constant under the constraint $\mathbf{b}^{\dagger} \mathbf{s}=1$ ). Thus the MVDR beamformer maximizes the signal to noise-plus-interference ratio. The result of this optimization is the optimal receiving beamformer [15]

$$
\mathbf{b}=\frac{\mathbf{R}^{-1} \mathbf{s}}{\mathbf{s}^{\dagger} \mathbf{R}^{-1} \mathbf{s}}
$$

Figure 5 shows plots of the transmitting beamformer $H\left(e^{j N_{r} \omega}\right)$, the receiving beamformer $G\left(e^{j \omega}\right)$, and the transmit-receive beam $H\left(e^{j N_{r} \omega}\right) G\left(e^{j \omega}\right)$. The vertical line in the middle represents target location and the dashed lines represent jammers. Note that $H\left(e^{j N_{r} \omega}\right)$ has many sharp mainlobes, of which $G\left(e^{j \omega}\right)$ chooses one main lobe which survives in the overall beam $H\left(e^{j N_{r} \omega}\right) G\left(e^{j \omega}\right)$. The effective beam indeed has a very sharp main lobe, with some noticeable bumps in the sidelobes representing suppressed grating lobes.

Example 2. One problem with an excessively sharp main beam is that if there is a mismatch between the actual target location and the assumed target location then the effective beam does not see the target. In this example we will present an IFIR SIMO radar design which is more robust to such a steering vector mismatch. We assume the target location is $1^{\circ}$ instead of $0^{\circ}$. We assume the transmitter does not know about this mismatch. The receiver however knows about the target location and designs an appropriate MVDR beamformer. We take $N_{t}=11$ and $N_{r}=10$, with $d_{t}=N_{r} d_{r}$ and $d_{r}=\lambda / 2$. If the transmitter sends the usual IFIR beam with a very narrow main lobe centered at $\theta=0^{\circ}$ as in the previous example, then the target would almost be missed (see Fig. 6). The trick in these situations is to design a transmitting beamformer with a flatter main lobe gain as shown in Fig. 7 (top) so that the transmit-receive beam is as in the bottom. This does indeed have nearly unity gain at the location of the target.

The flat transmitter design in our example is acheived using the filter design method in Sec. II of [14]. To be more specific, we first design $H_{3}(z)=H_{1}(z) H_{2}(z)$ where $H_{2}(z)=\left[\left(1+z^{-1}\right) / 2\right]^{3}$, and $H_{1}(z)$ is a 7 th order McClellanParks lowpass filter such that the cascade $H_{3}(z)$ has nearly equiripple passband. Note that $H_{3}(z)$ is a linear phase (Type 1) lowpass filter of order ten.
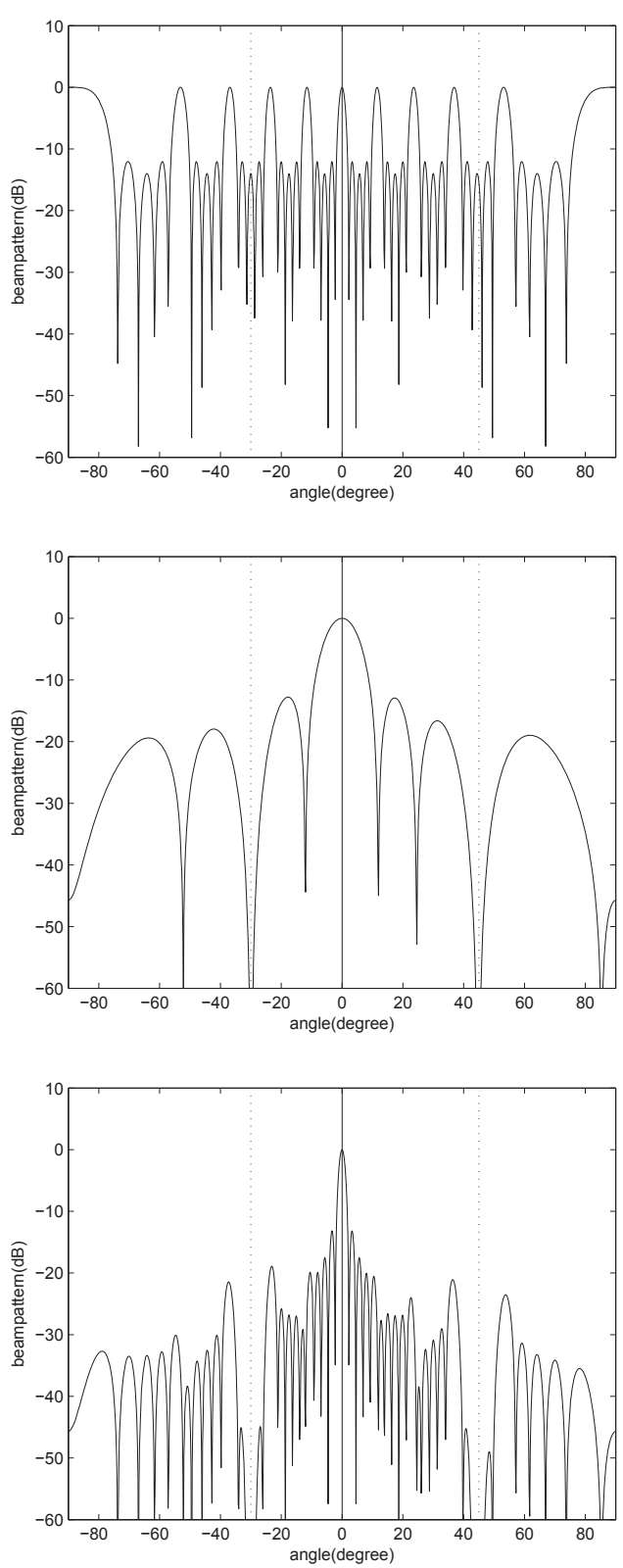

Fig. 5. Example 1. The FIR beamformer. Transmitter beam $H\left(e^{j \omega N_{r}}\right)$ (top), receiver beam $G\left(e^{j \omega}\right)$ (middle), and transmit-receive beam (bottom).

Since $z^{-5}-H_{3}(z)$ is a highpass filter, we can now define a lowpass filter

$$
H(z)=(-z)^{-5}-H_{3}(-z) .
$$

This is a 10th order lowpass filter, with a very flat passband. The passband flatness arises from the flatness of $H_{2}(z)$ at $\omega=\pi$, as explained in detail in [14]. The eleven coefficients of $H(z)$ are used as the weights in the transmitting beamformer. We use $d_{t}=N_{r} d_{r}$ with $d_{r}=\lambda / 2$, so that the transmitting beam is $H\left(z^{N_{r}}\right)$ (where $N_{r}=10$ ). Fig. 7 shows the plots of all relevant beams. Notice that owing to the passband flatness of the transmitting beam, the cascade $H\left(z^{10}\right) G(z)$ still has nearly peak gain at the target location $\theta=1^{\circ}$. In this example 
the receiver beam was designed using the MVDR appoach as in Ex. 1 (with the jammer and noise details precisely as in Ex. 1).

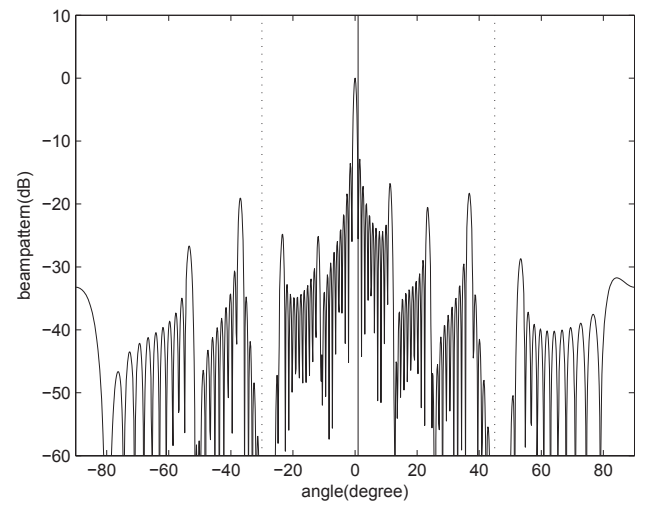

Fig. 6. Case where the IFIR transmit-receive beamformer misses a mismatched target owing to extreme sharpness of the beam.

\section{CONCLUDING REMARKS}

It was shown in [11] that the signal to clutter ratio of the IFIR radar is intermediate between that of the SIMO and MIMO radars (if the latter admits extra integration on target which in theory should be possible). In this paper however we have not considered clutter in the selection of the weights $\left\{a_{n}\right\}$ and $\left\{b_{n}\right\}$. The receiver coefficients $\left\{b_{n}\right\}$ were optimized for jammers but the transmitter coefficients $\left\{a_{n}\right\}$ were not optimized. An interesting practical problem would be to take clutter into account and jointly optimize $\left\{a_{n}\right\}$ and $\left\{b_{n}\right\}$ such that a linear combination of the signal to clutter ratio and signal to jammer ratio is maximized.

\section{REFERENCES}

[1] D. W. Bliss and K. W. Forsythe, "Multiple-input multiple-output radar and imaging: degrees of freedom and resolution," 37th Asilomar Conf. on Sig., Sys., and Comp., pp. 54-59, Nov. 2003.

[2] K. W. Forsythe, D. W. Bliss, and G. S. Fawcett, "MIMO Radar: performance issues," 38th Asilomar Conf. on Sig., Sys., and Comp., pp. 310-315, Nov. 2004.

[3] F. C. Robey, S. Coutts, D. Weikle, J. C. McHarg, and K. Cuomo, "MIMO Radar Theory and Experimental Results," 38th Asilomar Conf. on Sig., Sys., and Comp., pp. 300-304, Nov. 2004.

[4] E. Fishler, A. Haimovich, R. S. Blum, L. J. Cimini, D. Chizhik, and R. A. Valenzuela, "Performance of MIMO radar systems: Advantages of angular diversity," in Proc. IEEE Asilomar Conf. Sig., Sys., and Comp., pp. 305-309, Nov. 2004.

[5] J. Li, and P. Stoica, "MIMO radar with colocated antennas", IEEE Sig. Proc. Mag., pp. 106-114, Sept. 2007.

[6] A. M. Haimovich, R. S. Blum, and L. J. Cimini, Jr., "MIMO Radar with widely separated antennas", IEEE Sig. Proc. Mag., pp. 116-129, Jan. 2008.

[7] Chun-Yang Chen and P. P. Vaidyanathan, "MIMO radar space-time adaptive processing using prolate spheroidal wave functions", IEEE Trans. Signal Processing, pp. 623-635, Feb., 2008.

[8] F. Daum and J. Huang, "MIMO radar: snake oil or good idea?", Proc. IEEE Asil. Conf. on Sig., Sys., and Comp., Monterery, CA, Nov. 2008.

[9] A. Hassanien and S. A. Vorobyov, "Transmit/receive beamforming for MIMO radar with colocated antennas", Proc. IEEE Intl. Conf. Acoust., Speech, and Signal Proc., Taipei, 2009.

[10] J. Li and P. Stoica (editors), MIMO radar signal processing, John Wiley \& Sons, 2009
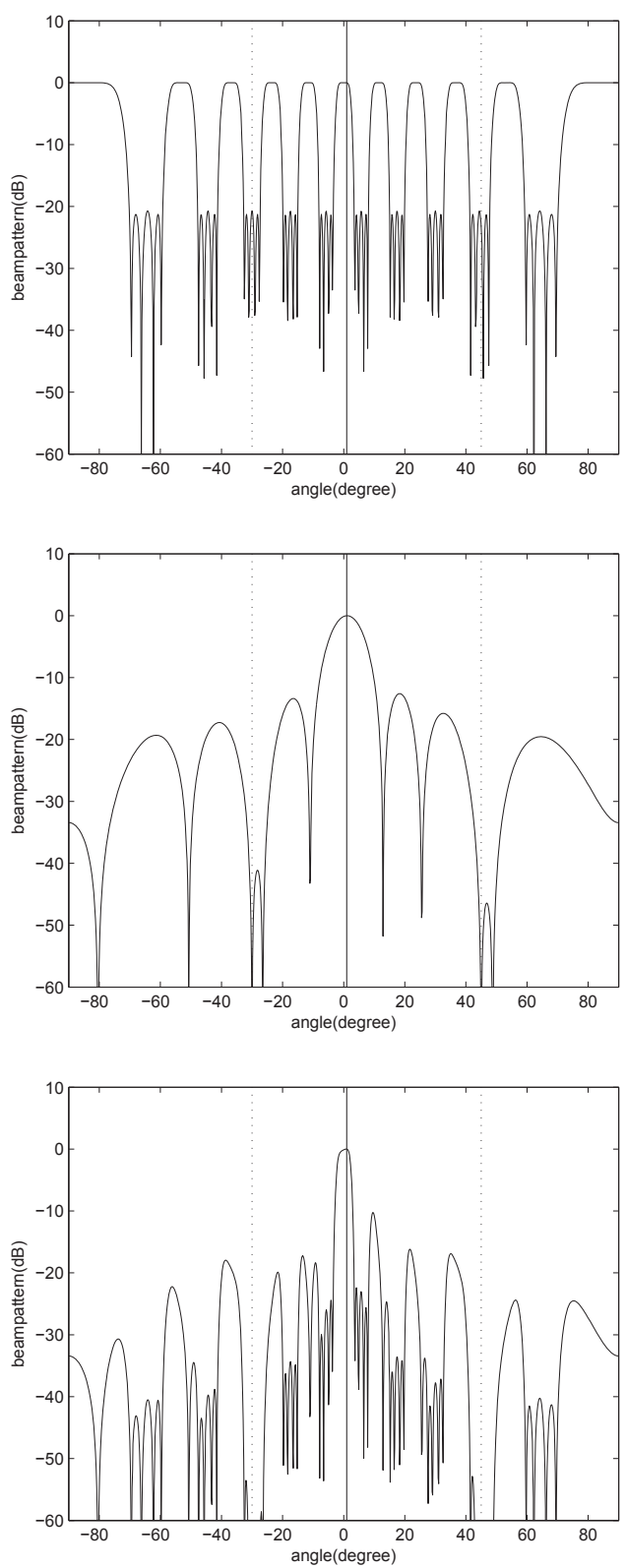

Fig. 7. Example 2. The FIR beamformer. Transmitter beam with improved flatness (top), receiver beam (middle) and transmit-receive beam (bottom).

[11] P. P. Vaidyanathan and Piya Pal, "MIMO radar, SIMO radar, and IFIR radar: a comparison," Proc. IEEE Asil. Conf. on Sig., Sys., and Comp., Monterery, CA, Nov. 2009 (preview copy at http://systems.caltech.edu/cweng/A2009.pdf).

[12] Y. Neuvo, C.-Y. Dong, and S. K. Mitra, "Interpolated finite impulse response filters", IEEE Trans. Acoust., Speech, and Signal Processing, pp. 563-570, June 1984.

[13] P. P. Vaidyanathan, Multirate systems and filter banks, Englewood Cliffs, NJ: Prentice-Hall, 1993. N. Y., 2002.

[14] P. P. Vaidyanathan, "Optimal design of linear-phase FIR digital filters with very flat passbands and equiripple stop bands", IEEE Trans. Circuits and System, pp. 904-917, Sept., 1985.

[15] H. L. Van Trees, Optimum array processing, John Wiley \& Sons, N. Y., 2002. 